\title{
AN INDEX FORMULA FOR AN $n$-TUPLE OF SHIFTS ON THE POLYDISK
}

\author{
KEREN YAN
}

(Communicated by Palle E. T. Jorgensen)

\begin{abstract}
Let $\left(M_{z_{1}}, \ldots, M_{z_{n}}\right)$ be an $n$-tuple of shift operators on the polydisk $l^{2}\left(\mathbf{Z}^{n}\right)$; we compress it to a variety of subspaces of $l^{2}\left(\mathbf{Z}^{n}\right)$ that are combinatorially constructed. The main result is a multivariate Fredholm index formula, which links the indices of the $n$-tuples to their combinatorial data in the definitions of the subspaces.
\end{abstract}

In [11], Taylor, using homological algebraic methods, introduced a natural spectral theory for $n$-tuples of commuting operators acting on Banach spaces. Fredholm theory and index theory are developed with respect to this spectral theory $[2,3,8]$. While this several variable spectral theory is a natural generalization of the classical single operator theory (when $n=1$ ), the techniques of computing these spectra are so different from or, much more complicated than, that of single operators.

The problem of deciding the spectral pictures of various classes of $n$-tuples of operators is very essential in multivariate operator theory and has deep applications to many areas of analysis, such as function theory on domains in $\mathbf{C}^{n}$. Many examples have been investigated through many different techniques $[4,5$, $7,9,10]$. In this note, we use the homological method to compute the spectra and Fredholm indices of a new, interesting class of $n$-tuples of operators. We will give an index formula for these $n$-tuples in terms of combinatorial data in their definition. An interesting consequence of this is that some combinatorial property of the operator forces the index to be nonzero.

To be more precise, we denote by $T=\left(T_{1}, \ldots, T_{n}\right)$ an $n$-tuple of commuting operators on the Hilbert space $H$ and by $K(T, z)=\left\{K^{q}(T, z), \partial^{q}\right\}$ the Koszul complex of $T$ at $z \in \mathbf{C}^{n}$, where $K^{q}(T, z)$ is the space of $q$-cochains and $\partial^{q}$ is the differential map at degree $q$. We refer the reader to Taylor's original papers $[11,12]$ for a more detailed account of these concepts. Recall that a point $z$ in $C^{n}$ is said to be in the Taylor spectrum $\sigma(T)$, if the Koszul

Received by the editors October 6, 1992.

1991 Mathematics Subject Classification. Primary 47D25.

Research supported in part by a grant from NSF, DMS 90-02969. 
complex $K(T, z)$ is not exact. The cohomology groups of the Koszul complex $K(T, z)=\left\{K^{q}(T, z), \partial^{q}\right\}$ is denoted by $H^{q}(T, z)$. The essential spectrum of $T$ is defined to be the set of points in $\mathbf{C}^{n}$ such that at least one of the groups $H^{q}(T, z)$ is infinite dimensional. A point $z$ is a Fredholm point for $T$, if all $H^{q}(T, z), 0 \leq q \leq n$, are finite-dimensional spaces [11, 12]. In this case, an index can be defined

$$
\operatorname{ind}(T-z)=\sum_{i=0}^{n}(-1)^{i} \operatorname{dim} H^{i}(T, z) .
$$

Now we define a special class of $n$-tuples of operators which is the main object of study in this paper.

Let $l^{2}(\mathbf{Z})$ be the little $l^{2}$ space on the integers $\mathbf{Z}, P$ the orthogonal projection onto the nonnegative part of $l^{2}$, e.g., $P l^{2}(\mathbf{Z})=\left\{f \mid f \in l^{2}(\mathbf{Z}), f(n)=0\right.$, $n<0\}$, and $Q=1-P$. Define

$$
H=l^{2}(\mathbf{Z}) \otimes \cdots \otimes l^{2}(\mathbf{Z})=l^{2}\left(\mathbf{Z}^{n}\right) .
$$

There is an orthogonal decomposition of $H$

$$
H=\sum_{\left(a_{1}, \ldots, a_{n}\right) \in N} \bigvee\left(a_{1}, \ldots, a_{n}\right),
$$

where $\bigvee\left(a_{1}, \ldots, a_{n}\right)=a_{1} \otimes \cdots \otimes a_{n}$ on $H$ and

$$
N=\left\{\left(a_{1}, \ldots, a_{n}\right) \mid a_{i}=P \text { or } Q\right\} .
$$

Apparently, $N$ is a finite set with $2^{n}$ elements, and each $\bigvee\left(a_{1}, \ldots, a_{n}\right)$ represents a subspace of $H$ supported on a conner of $\mathbf{Z}^{n}$. For example, if $n=2$, $H$ is simply the $l^{2}$ space of the plane lattice $\mathrm{Z}^{2}$ and each $\bigvee(\underline{a})$ represents a quadrant of the plane.

We denote by $u$ the bilateral shift operator on $l^{2}(\mathbf{Z})$ and define

$$
u_{i}=1 \otimes \cdots \otimes u \otimes \cdots \otimes 1, \quad i=1, \ldots, n,
$$

on $H .\left(u_{1}, \ldots, u_{n}\right)$ is an $n$-tuple commuting unitary operators on $H$.

Let $L \subset N$ be a subset of $N$. We define for each $j$ an action on $L$.

$$
A_{j}^{L}\left(a_{1}, \ldots, a_{n}\right)=\left\{\begin{aligned}
&\left(a_{1}, \ldots, a_{j-1}, P, a_{j+1}, \ldots, a_{n}\right) \\
& \text { if }\left(a_{1}, \ldots, a_{j-1}, P, a_{j+1}, \ldots, a_{n}\right) \in L, \\
&\left(a_{1}, \ldots, a_{n}\right) \quad \text { if }\left(a_{1}, \ldots, a_{j-1}, P, a_{j+1}, \ldots, a_{n}\right) \notin L .
\end{aligned}\right.
$$

Furthermore, we denote the space $\sum_{\underline{a} \in L} \bigvee(\underline{a})$ by $\bigvee(L)$.

Definition 1. $\underline{u}^{L}=\left(u_{1}^{L}, \ldots, u_{n}^{L}\right)$ is defined to be an $n$-tuple of operators acting on $\bigvee(L) H$, with

$$
u_{i}^{L}=\bigvee(L) u_{i} \bigvee(L) \mid \bigvee(L) H
$$

that is, $\underline{u}^{L}$ is the compression of the $n$-tuple of shifts to the space $\bigvee(L)$.

On the set $N$, we can define a degree function

$$
\begin{aligned}
& \operatorname{deg}: N \rightarrow \mathbf{Z}, \\
& \operatorname{deg}:\left(a_{1}, \ldots, a_{n}\right)=\left|a_{1}\right|+\cdots+\left|a_{n}\right|,
\end{aligned}
$$

where $|P|=1,|Q|=0$.

For each $L$, we denote by $L_{i}$ the set $L_{i}=\{\underline{a} \mid \underline{a} \in L, \operatorname{deg}(\underline{a})=i\}$, and by $\left|L_{i}\right|$ the cardinal of $L_{i}$.

Now we are in the position to state our main result. 
Theorem 1. Let $L \subset N$ such that $\underline{u}^{L}=\left(u_{1}^{L}, \ldots, u_{n}^{L}\right)$ is an $n$-tuple of commuting operators. Then every point $\underline{\lambda}$ in the polydisk $\mathbf{D}^{n}$ is a Fredholm point for $\underline{u}^{L}$ and

$$
\operatorname{ind}\left(\underline{u}^{L}-\lambda\right)=\sum_{i=1}^{n}(-1)^{i}\left|L_{i}\right| .
$$

This relates the index of $\underline{u}^{L}-\lambda$ and the combinatorial data $\left|L_{i}\right|$.

As an interesting corollary, we have

Corollary 1. If $|L|$ is odd, then ind $\left.\underline{u}^{L}-\lambda\right)$ is odd and therefore nonzero. In this case, $\sigma\left(\underline{u}^{L}\right)=\overline{\mathbf{D}}^{n}$ and $\sigma_{e}\left(\underline{u}^{L}\right)=\partial \overline{\mathbf{D}}^{n}$.

In $\S 2$ we give the proof of the above results, and in $\S 3$ we will discuss how to compute each cohomology group $H^{q}\left(\underline{u}^{L}-\lambda\right)$ by reducing the problem to the graph theory.

In this section, we first prove several lemmas that illustrate the general methods to handle the operator $n$-tuples $\underline{u}^{L}-\lambda$, and then, using these lemmas, we prove Theorem 1.

Lemma 1. Let $L \subset N . \underline{u}^{L}$ is an n-tuple of commuting operators if and only if, for each $\underline{a} \in L$ and $i, j$, either $A_{i}^{L} A_{j}^{L}=A_{j}^{L} A_{i}^{L}$ or $A_{i}^{L} A_{j}^{L}=A_{j}^{L}$ and $A_{j}^{L} A_{i}^{L}=A_{i}^{L}$.

Proof. By direct check and the definition of $\underline{u}^{L}$.

Remark. Not all $\underline{u}^{L}$ are commuting. For example, if $n=2$ and $L=\{(Q, P)$, $(P, P),(Q, Q)\}$, then $u^{L}$ is not commuting.

Lemma 2. If $\underline{u}^{L}$ is commuting and $L_{0} \subset L$ is an $\left\{A_{i}^{L}\right\}$-invariant subset for all $i$, then $\underline{u}^{L_{0}}$ is also commuting and, in this case $\underline{u}^{L-L_{0}}$ is also commuting.

Proof. Since $u_{i}^{L} \bigvee\left(a_{1}, \ldots, a_{n}\right) \subset \bigvee\left(a_{1}, \ldots, a_{n}\right)+\bigvee\left(A_{i}^{L}\left(a_{1}, \ldots, a_{n}\right)\right)$, we see that, if $L_{0}$ is invariant under $\left\{A_{i}^{L}\right\}_{i=1}^{n}$, then $\bigvee\left(L_{0}\right)$ is an invariant subspace of $u_{i}^{L}$. Therefore, the restriction $\underline{u}^{L_{0}}$ is also a commuting $n$-tuple. In this case, $\underline{u}^{L}$ induces a commuting $n$-tuple on the quotient space

$$
\bigvee(L) / \bigvee\left(L_{0}\right)=\bigvee\left(L \backslash L_{0}\right)
$$

which is equal to $u^{L-L_{0}}$; therefore, $u^{L-L_{0}}$ is commuting.

Lemma 3. Let $L \subset N$ such that $\underline{u}^{L}$ is commuting and $L_{0} \subset L$ be an $\left\{A_{i}\right\}_{i=1^{-}}^{n}$ invariant subset. Then the short exact sequence

$$
0 \rightarrow \bigvee\left(L_{0}\right) \rightarrow \bigvee(L) \rightarrow \bigvee\left(L-L_{0}\right) \rightarrow 0
$$

induces a long exact sequence

$$
\begin{aligned}
\cdots & \rightarrow H^{p}\left(\underline{u}^{L_{0}}-\lambda\right) \rightarrow H^{p}\left(\underline{u}^{L}-\lambda\right) \\
& \rightarrow H^{p}\left(\underline{u}^{L-L_{0}}-\lambda\right) \rightarrow H^{p+1}\left(\underline{u}^{L_{0}}-\lambda\right) \rightarrow \cdots .
\end{aligned}
$$

Proof. This is exactly Lemma 1.5 from Taylor's paper [12]. 
Lemma 4. Let $L=\left\{\left(a_{1}, \ldots, a_{n}\right)\right\}$ be a singleton and $\operatorname{deg}\left(a_{1}, \ldots, a_{n}\right)=p$. Then

(a) $\underline{u}^{L}$ is commuting,

(b) $\operatorname{dim} H^{p}\left(\underline{u}^{L}-\lambda\right)=1$, for $\lambda \in \mathbf{D}^{n}$;

(c) $H^{q}\left(u^{L}-\lambda\right)=0$, for all $q \neq p, \lambda \in \mathbf{D}^{n}$.

Proof. Without loss of generality, we assume $a_{1}=a_{2}=\cdots=a_{p}=P$ and $a_{p+1}=\cdots=a_{n}=Q$. Then

$$
\begin{array}{ll}
u_{i}^{L}=1 \otimes \cdots \otimes \cup_{+} \otimes \cdots \otimes 1, & i \leq p, \\
u_{j}^{L}=1 \otimes \cdots \otimes \cup_{+}^{*} \otimes \cdots \otimes 1, & j \geq p+1,
\end{array}
$$

where $U_{+}$is the unilateral shift operation on $l^{2} \quad(Z \geq 0)$. The Koszul complex

$$
\begin{aligned}
K\left(\underline{u}^{L}-\lambda\right) \approx & K\left(\cup_{+}-\lambda_{1}\right) \otimes \cdots \otimes K\left(\cup_{+}-\lambda_{p}\right) \\
& \otimes K\left(\cup_{+}^{*}-\lambda_{p+1}\right) \otimes \cdots \otimes K\left(\cup_{+}^{*}-\lambda_{n}\right) .
\end{aligned}
$$

By the Künneth formula,

$$
H^{i}\left(u^{L}-\lambda\right)=\sum_{i_{1}+\cdots+i_{n}=i} H^{i_{1}}\left(\cup_{+}-\lambda_{1}\right) \otimes \cdots \otimes H^{i_{n}}\left(\cup_{+}^{*}-\lambda\right)
$$

and by the fact that

$$
\begin{array}{ll}
\operatorname{dim} H^{0}\left(\cup_{+}-\lambda_{i}\right)=0, & \operatorname{dim} H^{1}\left(\cup_{+}-\lambda_{i}\right)=1, \\
\operatorname{dim} H^{0}\left(\cup_{+}^{*}-\lambda_{i}\right)=1, & \operatorname{dim} H^{1}\left(\cup_{+}^{*}-\lambda_{i}\right)=0,
\end{array}
$$

we complete the proof.

Corollary 2. If $L=\left\{\underline{a}_{1}, \ldots, \underline{a}_{k}\right\}$ and $\operatorname{deg} \underline{a}_{i}=p, i=1, \ldots, k$, then

(a) $\underline{u}^{L}$ is commuting,

(b) $\operatorname{dim} H^{p}\left(\underline{u}^{L}-\lambda\right)=k, \lambda \in \mathbf{D}^{n}$;

(c) $H^{q}\left(\underline{u}^{L}-\lambda\right)=0, q \neq p, \lambda \in \mathbf{D}^{n}$.

Proof. Since $\vee\left(\underline{a}_{i}\right) u_{s} \vee\left(\underline{a}_{j}\right)=0$, for all $s, i \neq j$, this shows that

$$
\underline{u}^{L}=\bigoplus_{i=1}^{k} \underline{u}^{\left\{a_{i}\right\}}
$$

By Lemma 4, the result follows.

The next corollary proves the first part of Theorem 1.

Corollary 3. Let $L \subset N$ and $\underline{u}^{L}$ commute. Then $\mathbf{D}^{n}$ is contained in the Fredholm domain of $\underline{u}^{L}$.

Proof. We prove by induction on $m=\min \{\operatorname{deg} \underline{a} \mid \underline{a} \in L\}$ in the descending order.

If $m=n$, then $L=\{P \otimes \cdots \otimes P\}$ and $u^{L}$ is simply the multiplication by $\underline{z}$ operator $n$-tuples on the Hardy space $H^{2}\left(\mathbf{D}^{n}\right)$. By Lemma 4, every point in $\overline{\mathrm{D}}^{n}$ is Fredholm.

Suppose the result is true for $n \geq m \geq k$. We prove it for $m=k-1$, since the actions $\left\{A_{i}^{L}\right\}_{i=1}^{n}$ are nondecreasing in degree. $L \backslash L_{k-1}$ is invariant under $\left\{A_{i}^{L}\right\}_{i=1}^{n}$. By Lemma 2 and the induction hypothesis, $\underline{u}^{L-L_{k-1}}$ is Fredholm on $\mathbf{D}^{n}$. 
We have

$$
0 \rightarrow \bigvee\left(L \backslash L_{k-1}\right) \rightarrow \bigvee(L) \rightarrow \bigvee\left(L_{k-1}\right) \rightarrow 0,
$$

since $u^{L-L_{k-1}}$ is Fredholm and $\underline{u}^{L_{k-1}}$ is Fredholm, by Corollary 3 , on $\mathbf{D}^{n}$. Therefore, $\underline{u}^{L}$ is also Fredholm on $\mathbf{D}^{n}$.

Now we complete the proof of Theorem 1.

Proof of Theorem 1. If $\underline{u}^{L}$ is commuting and $L^{\prime}$ is an invariant subset of $L$, then the short exact sequence

$$
0 \rightarrow \bigvee\left(L^{\prime}\right) \rightarrow \bigvee(L) \rightarrow \bigvee\left(L \backslash L^{\prime}\right) \rightarrow 0
$$

induces a long exact sequence in cohomology groups

$$
\cdots \rightarrow H^{p}\left(L^{\prime}, \lambda\right) \rightarrow H^{p}(L, \lambda) \rightarrow H^{p}\left(L \backslash L^{\prime}, \lambda\right) \rightarrow H^{p+1}\left(L^{\prime}, \lambda\right) \rightarrow \cdots .
$$

It is a standard fact that the Euler numbers have the following relation:

$$
\sum_{i=1}^{n}(-1)^{i} \operatorname{dim} H^{i}(L, \lambda)=\sum_{i=1}^{n}(-1)^{i} \operatorname{dim} H^{i}\left(L^{\prime}, \lambda\right)+\sum_{i=1}^{n}(-1)^{i} \operatorname{dim} H^{i}\left(L \backslash L^{\prime}, \lambda\right) ;
$$

that is,

$$
\operatorname{index}\left(\underline{u}^{L^{\prime}}-\lambda\right)+\operatorname{index}\left(\underline{u}^{L \backslash L^{\prime}}-\lambda\right)=\operatorname{index}\left(\underline{u}^{L}-\lambda\right) \text {. }
$$

Now, for each $p, 0 \leq p \leq n$, we have the short exact sequence

$$
0 \rightarrow \bigvee\left(L_{p}\right) \rightarrow \bigvee\left(L \backslash \bigcup_{i>p} L_{i}\right) \rightarrow \bigvee\left(L \backslash \bigcup_{i \geq p} L_{i}\right) \rightarrow 0
$$

This yields that, for each $p$, we have

$$
\operatorname{ind}\left(u^{L_{p}}-\lambda\right)=\operatorname{ind}\left((\underline{u})^{L \backslash \bigcup_{i>p} L_{i}}-\lambda\right)-\operatorname{ind}\left((\underline{u})^{L \backslash \bigcup_{i \geq p} L_{i}}-\lambda\right) .
$$

Adding all equations together, we have

$$
\sum_{p=0}^{n} \operatorname{ind}\left(u^{L_{p}}-\lambda\right)=\operatorname{ind}\left(\underline{u}^{L}-\lambda\right) .
$$

Note that $\operatorname{ind}\left(u^{L_{p}}-\lambda\right)=(-1)^{p}\left|L_{p}\right|$. The proof is thus complete.

3

In this section, we discuss how to determine the cohomology groups $H^{q}\left(\underline{u}^{L}-\lambda\right)$. We first give a graph-theoretic representation of the operators $\underline{u}^{L}$ and then show how certain graph operations can be used to determine the cohomology groups.

From the set $N$, we define a directed graph $\widehat{N}$. The vertices of $\widehat{N}$ are simply points of $N$. If $\underline{a}, \underline{b}$ are two distinct points in $N$ and $A_{j}^{N} \underline{a}=\underline{b}$ for some $j$, then there is an arrow $\underline{a} \underline{b}$ from $\underline{a}$ to $\underline{b}$ in $\widehat{N} . \widehat{N}$ is the graph with $N$ as the set of vertices and the arrows defined above. If $L$ is a subset of $N$, we define a subgraph $\widehat{L}$ of $\widehat{N}$ by

$$
\text { vertices of } \widehat{L}=L, \quad \text { arrows of } \widehat{L}=\{\underline{\vec{a}} \underline{\vec{b}} \in \widehat{N} \mid \underline{a}, \underline{b} \in L\} .
$$


If $L^{\prime} \subset L$, we call $L^{\prime}$ invariant in $L$, if every arrow in $\widehat{L}$ that starts from $L^{\prime}$ also ends in $L^{\prime}$; we call $L^{\prime}$ coinvariant in $L$, if $L \backslash L^{\prime}$ is invariant in $L$. In graph theory language, invariant is called absorbent; coinvariant is called inaccessible.

The following facts are easily checked.

(1) $L^{\prime} \subset L$ is invariant in $L$ if and only if $L^{\prime}$ is $\left\{A_{i}^{L}\right\}_{i=1}^{n}$-invariant.

(2) If $\underline{\vec{a} \underline{b}}$ is an arrow in $\widehat{N}, A_{j}^{N} \underline{a}=\underline{b}$, and $L=\{\underline{a}, \underline{b}\}$, then $\sigma\left(\underline{u}^{L}\right)=$ $\overline{\mathbf{D}} \times \cdots \times \overline{\mathbf{D}} \times \partial \mathbf{D} \times \overline{\mathbf{D}} \times \cdots \times \overline{\mathbf{D}}$.

Consequently, if $\lambda=\left(\lambda_{1}, \ldots, \lambda_{n}\right)$ and $\left|\lambda_{j}\right| \neq 1$, then

$$
H^{q}\left(\underline{u}^{L}-\lambda\right)=0 \text { for all } q .
$$

(3) $L \subset N$. If $\widehat{L}$ has no arrow, then $\underline{u}^{L}=\bigoplus_{\underline{a} \in L} u^{\{\underline{a}\}}$.

Lemma 5. If $\underline{u}^{L}$ is commuting, then $\partial \overline{\mathbf{D}^{n}} \subset \sigma\left(\underline{u}^{L}\right)$.

Proof. Let $k=\max \{\operatorname{deg} \underline{a} \in L\}$ and $\underline{a} \in L, \operatorname{deg} \underline{a}=k$.

$$
u^{L} \mid \bigvee(\underline{a}) \cong\left(U_{+} \otimes \cdots \otimes 1, \ldots, 1 \otimes \cdots \otimes \cup_{+}^{*}\right) \text {. }
$$

For each $\lambda \in \partial \overline{\mathbf{D}}^{n}$, we can find $f_{j}^{i} \in l^{2} \quad(\mathbf{Z} \geq 0)$ such that

$$
\left\|\left(u_{i}^{L}-\lambda_{i}\right) f_{j}^{i}\right\| \rightarrow 0 \quad \text { as } j \rightarrow \infty \text { and }\left\|f_{j}^{i}\right\|=1 .
$$

This shows either $H^{0}\left(u^{L}-\lambda\right) \neq 0$ or $H^{1}\left(u^{L}-\lambda\right) \neq 0$.

The following lemma is most important in computing Taylor spectra.

Lemma 6. Let $L \subset N$ such that $\underline{u}^{L}$ is commuting, and let $\underline{a} \vec{b}$ be an arrow in $\widehat{L}$ such that $A_{j}^{N} \underline{a}=\underline{b}$. If $\{\underline{a}, \underline{b}\}$ is invariant or coinvariant in $L$, then, for each $\lambda=\left(\lambda_{1}, \ldots, \lambda_{n}\right),\left|\lambda_{j}\right| \neq 1$, we have $H^{q}\left(\underline{u}^{L}-\lambda\right)=H^{q}\left(\underline{u}^{L} \backslash\{\underline{a}, \underline{b}\}-\lambda\right)$ for all $q$. Proof. If $\{\underline{a}, \underline{b}\}$ is invariant, then we have the following short exact sequence

$$
0 \rightarrow \bigvee(\{\underline{a}, \underline{b}\}) \rightarrow \bigvee(L) \rightarrow \bigvee(L \backslash\{\underline{a}, \underline{b}\}) \rightarrow 0
$$

In the induced long exact sequence, we notice that $H^{q}\left(u^{\{a, b\}}-\lambda\right)=0$ for all $q$. Therefore, $H^{q}\left(\underline{a}^{L}-\lambda\right)=H^{q}\left(u^{L \backslash\{\underline{a}, \underline{b}\}}-\lambda\right)$ for all $q$. The case of $\{\underline{a}, \underline{b}\}$ coinvariant is proved similarly, only replacing the short exact sequence by

$$
0 \rightarrow \bigvee(L \backslash\{\underline{a}, \underline{b}\}) \rightarrow \bigvee(L) \rightarrow \bigvee(\{\underline{a}, \underline{b}\}) \rightarrow 0
$$

Lemma 6 enables us to reduce the computation of $H^{q}\left(\underline{u}^{L}-\lambda\right)$ to that of a smaller graph $L \backslash\{\underline{a}, \underline{b}\}$. If we can keep doing this process, we will reduce the graph $\widehat{L}$ to a graph of $\widehat{L}^{\prime}$ which contains no arrow. Then (3) applies finally to compute the cohomology groups. But, in general, we do not know whether this procedure can always continue. However, for many examples, this method does provide a powerful tool of computation. We finish this paper by giving several examples.

Example 1. $L=\{\underline{a} \mid \operatorname{deg} \underline{a} \geq n-1\}$. In this case, $L$ consists of $n+1$ elements, $P \otimes \cdots \otimes P, Q \otimes P \otimes \cdots \otimes P, \ldots, P \otimes \cdots P \otimes Q$. It is easily seen that $L$ is $\left\{A_{i}^{N}\right\}$-invariant, so $\underline{u}^{L}$ is a restriction of $\underline{u}^{N}$ and thus an $n$-tuple of joint sub- 
normal operators. Its graph is shown as

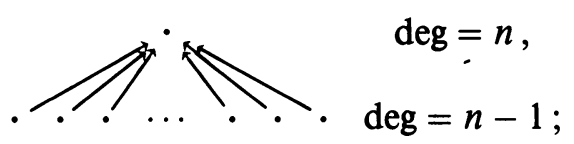

each arrow is invariant. If we take off one arrow, say the left arrow, then the remaining graph $L^{\prime}$ becomes:

o

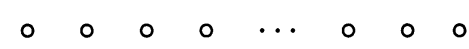

It is a graph without arrows.

Suppose $\lambda=\left(\lambda_{1}, \ldots, \lambda_{n}\right) \in \mathbf{D}^{n}$. Then each arrow can be taken off by Lemma 6, and $H^{q}\left(\underline{u}^{L}-\lambda\right)=H^{q}\left(\underline{u}^{L}-\lambda\right)=H^{q}\left(\bigoplus_{\underline{a} \in L^{\prime}} u^{\{\underline{a}\}}-\lambda\right)$. Thus

$$
H^{q}\left(\underline{u}^{L}-\lambda\right)= \begin{cases}\mathbf{C}^{n-1}, & q=n-1, \\ 0, & \text { otherwise. }\end{cases}
$$

If $n \neq 1 \quad\left(n=1\right.$ is easy), we have $\sigma\left(\underline{u}^{L}\right)=\overline{\mathbf{D}}^{n}$ and $\sigma_{e}\left(\overline{\mathbf{D}}^{n}\right)=\partial \overline{\mathbf{D}}^{n}$. This example also justifies our index formula index $\left(\underline{u}^{L}-\lambda\right)=(-1)^{n-1}(n-1)=$ $\sum_{i=0}^{n}(-1)^{i}\left|L_{i}\right|$.

Example 2. $n=3$ and $L=\{\underline{a} \mid \operatorname{deg} \underline{a} \geq 1\}$.

$L$ has the graph

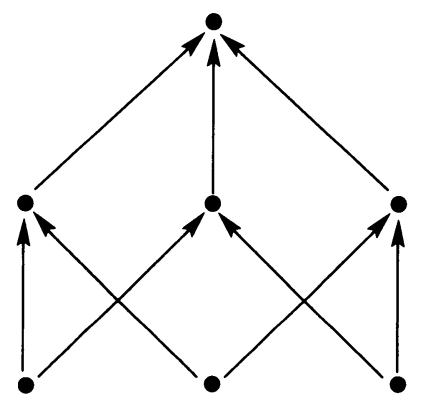

By successively taking off invariant arrows, as given by the following steps:

○

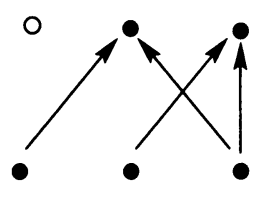

Step 1
O

O

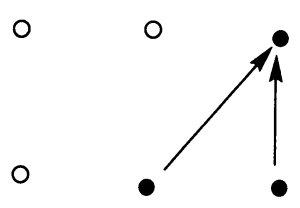

Step 2
O

O

O

Step 3

we get only one point left which is degree 1 . 
Therefore, if $\lambda \in \mathbf{D}^{n}$,

$$
H^{q}\left(\underline{u}^{L}-\lambda\right)= \begin{cases}1, & q=1 \\ 0, & q \neq 1 .\end{cases}
$$

Consequently, $\sigma\left(u^{L}\right)=\overline{\mathbf{D}}^{3}$, and $\sigma_{e}\left(u^{L}\right)=\partial \overline{\mathbf{D}}^{3}$.

The techniques used in the examples show that we can construct joint subnormal $n$-tuples with all kinds of prescribed cohomology groups $H^{q}\left(u^{L}\right)$. This provides a large class of operator $n$-tuples which do not have property $(\beta)$ [7]. Moreover, since the polydisk $\mathbf{D}^{n}$ is the Fredholm domain of these operator $n$ tuples, the cohomology sheaves of the Koszul complex are all Hermitian vector bundles on $\mathbf{D}^{n}$. Using the Hermitian sheaf theory [13], one can obtain unitary invariants of these operators; that will be treated elsewhere.

\section{REFERENCES}

1. J. B. Conway, Subnormal operators, Res. Notes in Math., vol. 51, Pitman, New York, 1981.

2. R. E. Curto, Fredholm and invertible n-tuple of operators, The transformation problem, Trans. Amer. Math. Soc. 266 (1981), 129-159.

3. __ Spectral permanence for joint spectra, Trans. Amer. Math. Soc. 270 (1982), 659-665.

4. R. E. Curto and K. Yan, Spectral theory of Reinhardt measures, Bull. Amer. Math. Soc. (N.S.) 24 (1991), 379-385.

5. __ The Taylor spectrum of infinite direct sums, Contemp. Math., vol. 120, Amer. Math. Soc., Providence, RI, 1991, pp. 19-27.

6. R. G. Douglas and V. I. Paulsen, Hilbert modules over function algebras, Pitman Res. Notes in Math., vol. 127, Longman Sci. Tech., Harlow, 1989.

7. M. Putinar, Spectral theory and sheaf theory. II, Math. Z. 192 (1986), 473-490.

8. 674-692.

9. N. Salinas, The $\bar{\partial}$-Fredholm and the $C^{*}$-algebra of the Bergman $n$-tuple, J. Operator Theory 22 (1989), 325-343.

10. N. Salinas, A. Sheu, and H. Upmier, Toeplitz operators on pseudo-convex domains and foliation $C^{*}$-algebras, Ann. of Math. (2) 130 (1989), 531-565.

11. J. Taylor, A joint spectrum of several commuting operators, J. Funct. Anal. 6 (1970), 172-191.

12. (1970), 1-38.

13. K. Yan, Hermitian sheaves and operator theory, preprint, 1992.

Department of Mathematical SCIEnces, Indiana University-PuRdue University at INDiANAPOLIS, 1125 EAST 38Th STREET, INDIANAPOLIS, INDIANA 46205-2810 